\title{
COVID-19 ACTUALIZACION. Nueva variante, nuevas terapias, vacunas para niños
}

\author{
Davis Gaus[1]
}

1. Andean Health \& Development, Estados Unidos.

Doi: https://doi.org/10.23936/pfr.v6i3.214

PRÁCTICA FAMILIAR RURAL | Vol.6 | No.3 | Noviembre 2021 | Recibido: 30/10/2021 | Aprobado: 20/11/2021

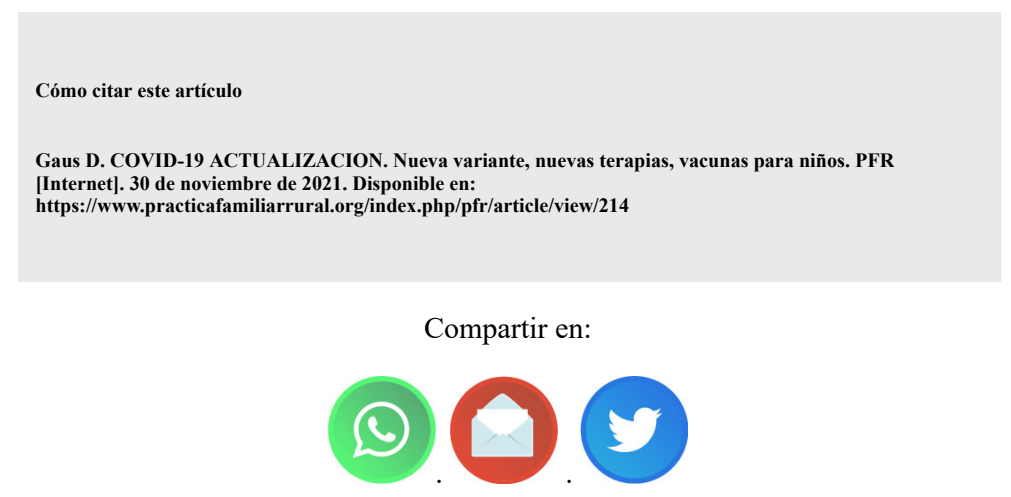

\section{Resumen}

Se realiza una actualización del conocimiento medico científico sobre el COVID-19 en relación, a la epidemiologia, factores de riesgo y comorbilidades, fisiopatología, utilidad de los exámenes de imagen, tratamiento, y vacunas.

Palabras clave:Covid-19, actualización noviembre 2021, guía clínica

COVID-19 UPDATE. New variant, new therapies, vaccines for children

\begin{abstract}
An update of the scientific medical knowledge about COVID-19 in relation to epidemiology, risk factors and comorbidities, pathophysiology, usefulness of imaging tests, treatment, and vaccines is carried out.
\end{abstract}

Key words:Covid-19, Nov 2021 update, clinical guidelines

\section{Variante Omicrón}

Suráfrica reporto el 24 noviembre de 2021 la existencia de una nueva variante de COVID-19 que nombraron Omicrón. Cuenta con 50 mutaciones, de los cuales 30 están ubicadas en la Proteína S.1 La evidencia preliminar sugiere que la mayoría de los sitios que sirven como "un blanco" para los anticuerpos generados por las vacunas han mutado. 2

Los datos preliminares de la virulencia de la nueva cepa, provoca preocupación en la comunidad científica, sobre el posible aumento de la transmisibilidad del virus. Epidemiológicamente, el descubrimiento de la variante también coincide con el crecimiento de casos en Suráfrica en los últimos meses del 2021. Existe una preocupación por la virulencia de la nueva variante. Necesitamos nuevos datos sobre la severidad clínica. Tampoco existen datos sobre el impacto de las vacunas sobre transmisión de la variante Omicrón ni de la eficacia para prevenir complicaciones en pacientes infectados con esta nueva variante.

Además de felicitar a Sudáfrica por su sistema de vigilancia epidemiológica, y el descubrimiento temprano de la nueva variante, la comunidad global opto' por aislar a 6 paises en el sur de África con la intención de contener su transmisión. Esta política de "castigo" ha sido criticada por muchas lideres mundiales, tomando en cuenta que la variante ha sido reportada en 5 continentes, demostrando que ya existe transmisión fuera de la región.

\section{Actualización de la Terapia Antiviral}

El nuevo fármaco antiviral, Molnupiravir, pertenece al grupo farmacéutico del remdesivir y Favipiravir, que inhiben la enzima RNA polimerasa (RdRp), su administración es oral lo cual es una ventaja frente al Remdesivir que solo se administra por vía intravenoso y Favipiravir que demuestra dificultades con su farmacocinética, que son desventajas importantes, frente al nuevo antiviral oral. $\underline{3}$ 
Molnupiravir se administra vía oral con un perfil farmacocinética favorable, un aclaramiento rápido, y demuestra una disminución rápida de la carga viral y una recuperación clínica rápida. 4 Demuestra pocos efectos secundarios y es bien tolerado.

Ensayos clínicos de fase 3 demuestran que molnupiravir redujo el riesgo por 50\% de hospitalización o la muerte cuando se les administra tempranamente a pacientes de alto riesgo de complicaciones. $\underline{5}$ Merck indica que la meta con el medicamento es administrarlo dentro de 24 horas del inicio de síntomas, y luego de una prueba positiva de COVID-19, que puede ser problemático en muchos contextos.

Se debe confirmar la dosis cuando esté disponible en el mercado. Sin embargo, la recomendación actual, en adultos es $800 \mathrm{mg}$ dos (2) veces al dia por cinco (5) días. El fármaco podría estar disponible hasta fines de 2021. $\underline{6}$

Para paises de bajos ingresos, la farmacéutica Merck permitiría la producción de molnupiravir en paises de África y Asia para disminuir los costos y hacerlo más disponible a nivel mundial..

Vacunas aprobadas para niños de 5-11 años

\section{PfizerNBiotech}

En noviembre del presente año, la FDA y el CDC estadounidense aprobaron con la calificación de "para uso de emergencia" la vacuna de Pfizer para niños entre 5-11 años. Los datos de Pfizer (aun no publicados) indica que la vacuna es segura y previene infección sintomática en $90.7 \%$ de niños de esta edad. 8 La vacuna neutraliza efectivamente la variante Delta.

La dosis de la vacuna de Pfizer es la décima parte de la dosis para adultos y la segunda dosis se debe administrar después de 21 días. Las autoridades de Pfizer dicen que la dosis busca un equilibrio entre obtener una respuesta inmune adecuada y disminuir los efectos secundarios.

No existe una "dosis por peso" de las vacunas, no se debe pensar en la vacuna como se piensa de otros fármacos. En el caso de una vacuna, se busca la dosis mínima para estimular al sistema linfático para que produzca anticuerpos. La determinación de la dosis de la vacuna se determinó en un ensayo clínico controlado.

Según la FDA, los niños entre 5-11 años representan el 9\% de todos los casos reportados en los Estados Unidos, y actualmente ese grupo etario demuestra la tasa mas alta de COVID que toda la población. 9 Salubristas en los EE UU reconocen que complicaciones graves de una infección de COVID-19 son menos frecuentes en niños que adultos, pero aun hay complicaciones graves en niños, como el síndrome inflamatorio multisistémico (MIS-C) y miocarditis.

En los ensayos clínicos de las vacunas en jóvenes entre 12-17 años, reportaron' miocarditis como efecto secundario poco frecuente (1 caso de cada 20,000 vacunadas) asociado a las vacunas de Pfizer y Moderna, especialmente en sexo masculino. La mayoría de las personas entre 16 a 29 años se recuperan completamente. Los ensayos clínicos de la vacuna de Pfizer en niños entre 5 a 11 años no demostraron ningún caso de miocarditis o ningún otro efecto adverso grave. Sin embargo, el tamaño del ensayo clínico es cuestionable, 2,400 participantes, un número insuficiente para detectar miocarditis.

La miocarditis como complicación de la infección por COVID-19 es más severa que la miocarditis asociada a la vacuna. Otros efectos secundarios menos graves de la vacuna en niños incluyen: adenopatía, cansancio, cefalea, y dolor en el sitio de la inoculación.

\section{Sinovac (Coronavac)}

Coronavac, que utiliza una tecnología (virus inactivado) para su vacuna muy distinta a la vacuna de Pfizer, fue aprobada para uso emergente en niños entre 3-17 años en China en junio de 2021, luego de ensayos clínicos de fase I/II. 10 Los ensayos demostraron la presencia de anticuerpos neutralizantes 28 días después de la segunda dosis. Los afectos adversos fueron de $26 \%$ de los participantes e incluyeron reacciones leves/moderadas dentro de 7 días de la administración de la vacuna. China esta' vacunando a los niños entre 3-17 años con Coronavac, y algunos países están usando esta vacuna en los últimos meses, incluyendo Cambodia, Ecuador, Chile, Argentina y Venezuela. Sinovac no esta' aprobada en los EE UU para niños. En el caso ecuatoriano Sinovac se utilizo para la vacunación de los niños.

Hasta la fecha, no existen estudios sobre el uso de otras vacunas de COVID-19 para niños entre 5-11 años.

Cuestionamiento a la evidencia sobre la eficacia de las vacunas COVID-19

La metodología utilizada para determinar la eficacia de las vacunas de COVID-19, en algunos círculos científicos, es cuestionada. El concepto del "efecto del usuario sano" fue descrito hace mas de 30 años y mantiene su presencia en la literatura.11 El efecto puede darse cuando el grupo de estudio (que recibe la intervención, como en este caso la vacuna de COVID-19) es demográficamente diferente que el grupo de control o placebo (que no recibe la intervención). Esa diferencia puede ser de varias categorías, como hábitos sanos, mejores condiciones de vida, mayor nivel educativo, mejores empleos, vivir en comunidades mas sanos, entre otras.

Si los dos grupos no son similares en todo, salvo la intervención (vacuna), el beneficio que confiere la vacuna puede ser confundido y por ende sobredimensionado por otros factores que los investigadores no tomaron en cuenta.

Recién se publicó un comentario de esta naturaleza sobre el monitoreo del impacto que las vacunas de COVID-19 tienen sobre mortalidad. Este artículo argumenta que las estadísticas sobre la protección que la vacuna de COVID ofrece contra la muerte, en el mundo real, están infladas y manipuladas, pues al comparar los grupos de vacunados versus los no-vacunados no se tomaron en cuenta factores como: edad, comorbilidades, obesidad, etc. citando el "efecto de usuario sano."

En el mismo articulo el autor cita un estudio en España del grupo de trabajo de la efectividad de la vacunación frente a COVID-19 realizada por el Ministerio de Sanidad Español, que demuestra que la eficacia de la vacuna disminuye luego de seis meses. "En el 
colectivo de personas residentes en centros de mayores, la efectividad se mantiene en valores superiores al $96 \%$ frente a infección, infección sintomática, hospitalización y defunción, en los vacunados de mayo en adelante. En los vacunados en marzo la efectividad disminuye a $58 \%, 64 \%, 65 \%$ y $77 \%$, para infección, infección sintomática, hospitalización y defunción, respectivamente, lo cual podría indicar una pérdida de inmunidad con el tiempo desde la vacunación.”12

Israel, un país que utiliza el "Pasaporte Covid" introdujo una normativa en octubre de 2021 requiriendo una tercera dosis de vacuna si ha pasado mas que 6 meses desde la segunda vacunación para no perder los privilegios del pasaporte. 13

Esta normativa a la que se van sumando muchos países, sumada a la posibilidad de que la protección que brindan las vacunas actuales puede disminuir paulatinamente a partir de los 6 meses, nos hace pensar en la posibilidad de que, a nivel global, los seres humanos necesitarían revacunarse cada seis meses. Tomando en cuenta que menos de la mitad del planeta esta completamente vacunado actualmente14, logísticamente se ve muy difícil que los países, logren encontrar una manera de vacunar a toda la población mundial cada seis meses.

Esta limitación provoca dudas sobre las expectativas que el mundo político y los medios de comunicación han levantado alrededor de la vacunación masiva y la reactivación económica. El mundo científico espera el desarrollo de nuevas vacunas que pueden brindar una protección por mayor tiempo.

\section{Referencias bibliográficas}

1. Browett A. Covid-19 Situation Report. Johns Hopkins Center for Health Security. Acceso 30 nov 2021. https://mail.google.com/mail/u/0/\#inbox/FMfcgzGllCmcNtTXkcgfrcBxxMVVfnWT

2. Browett A, Covid-19 Situation Report, 30 Nov 2021

3. Imran M, Arora M, Asdaq S, et al. Discovery, development, and patent trends on molnupiravir: a prospective oral treatment for COVID-19. Molecules. 2021， 26;5795. https://mdpi-res.com/molecules/molecules-26-05795/article_deploy/molecules-2605795.pdf

4. Fischer, W.; Eron, J.; Holman, W.; Cohen, M.S.; Fang, L.; Szewczyk, L.J.; Sheahan, T.P.; Baric, R.; Mollan, K.R.; Wolfe, C.R.; et al. Molnupiravir, an Oral Antiviral Treatment for COVID-19. medRxiv 2021.

5. Merck news release. Merck and Ridgeback's investigational oral antiviral molnupiravir reduced the risk of hospitalizacion or death by approximately 50 percent compared to placebo for pateints with mild or moderate COVID-19 in positive interim analysis of phase 3 study. Oct 1, 2021. https://www.merck.com/news/merck-and-ridgebacks-investigational-oral-antiviralmolnupiravir-reduced-the-risk-of-hospitalization-or-death-by-approximately-50-percent-compared-to-placebo-for-patients-withmild-or-moderat/

6. Robbins R. Merck says it has the first antiviral pill found to be effective against Covid. New York Times. 27 Oct 2021. https://www.nytimes.com/2021/10/01/business/covid-antiviral-pill-merck.html? campaign id $=154 \&$ emc $=$ edit cb $20211027 \&$ instance $\_\mathrm{id}=43967 \& \mathrm{nl}=$ coronavirusbriefing\&regi_id $=162851164 \&$ segment_id=72840\&te $=1 \&$ user_id $=9$ aa $1585711 \mathrm{e} 7638 \mathrm{a} 66 \mathrm{~b} 0 \mathrm{dab} 788 \mathrm{c} 44 \mathrm{~d} 8 \mathrm{e}$

7. Merck news release. Merck, Ridgeback Biotherapeutics and Emory University will not receive royalties for sales of molnupiravir under this agreement for as long as COVID-19 remains classified as a public health emergency of international concern by the WHO. 27 Oct 2021. https://www.merck.com/news/the-medicines-patent-pool-mpp-and-merck-enter-into-license-agreement-formolnupiravir-an-investigational-oral-antiviral-covid-19-medicine-to-increase-broad-access-in-low-and-middle-income-countri/

8. Hensley S. Pfizer-BioNTech COVID vaccine appears more than 90\% effective in kids 5 to 11 . NPR news. 22 oct 2021. https:/www.npr.org/sections/health-shots/2021/10/22/1048334791/pfizer-biontech-covid-vaccine-appears-more-than-90effective-in-kids-5-to-11

9. Neel J. 26 oct 2021

10. Han B, Song Y, Li C, et al. Safety, tolerability, and immunogenicity of an inactivated SARS-CoV-2 vaccines (Coronavac) in healthy children and adolescents: a double blind, randomized, controlled, phase $1 / 2$ clinical trial. The Lancet Inf Dis. Published on line 28 June 2021. DOI: https://doi.org/10.1016/S1473-3099(21)00319-4

11. Shrank W, Patrick A, Brookhart M. Healthy user and related biases in observational studies of preventive interventions: a primer for physicians. J Gen Intern Med. 2011 May;26(5):546-50 doi: 10.1007/s11606-010-1609-1.

12. Análisis de la efectividad de la vacunación frente a COVID-19 en España https://www.mscbs.gob.es/profesionales/saludPublica/prevPromocion/vacunaciones/covid19/docs/Efectividad VacunacionCOVID19 Espana.pdf

13. Kershner I. Israel will require a booster shot to be considered fully vaccinates. New York Times. 21 oct 2021. https://www.nytimes.com/2021/10/03/world/israel-covid-booster.html

14. Browett A. COVID-19 Situation Update. https://mail.google.com/mail/u/0/\#search/hopkis/FMfcgzGlkjcZzKBtsgTBlwHZVxwJsGbf

Hopkins. $\quad 4 \quad$ Nov 